\title{
Residues From Coal Conversion and Utilization: Advanced Mineralogical Characterization and Disposed Byproduct Diagenesis
}

\author{
Semi-Annual Report \\ December 1, 1996 - May 30, 1997 \\ By
Gregory J. McCarthy
}

Work Performed Under Contract No.: DE-FG22-96PC96207

For

U.S. Department of Energy

Office of Fossil Energy

Federal Energy Technology Center

P.O. Box 880

Morgantown, West Virginia 26507-0880

By

North Dakota State University

Department of Chemistry

Fargo, North Dakota 58102-5516 


\section{Disclaimer}

This report was prepared as an account of work sponsored by an agency of the United States Government. Neither the United States Government nor any agency thereof, nor any of their employees, makes any warranty, express or implied, or assumes any legal liability or responsibility for the accuracy, completeness, or usefulness of any information, apparatus, product, or process disclosed, or represents that its use would not infringe privately owned rights. Reference herein to any specific commercial product, process, or service by trade

name, trademark, manufacturer, or otherwise does not necessarily constitute or imply its endorsement, recommendation, or favoring by the United States Government or any agency thereof. The views and opinions of authors expressed herein do not necessarily state or reflect those of the United States Government or any agency thereof. 
RESIDUES FROM COAL CONVERSION AND UTILIZATION: ADVANCED MINERALOGICAL CHARACTERIZATION AND DISPOSED BYPRODUCT DIAGENESIS

DE-FG22-96PC96207--01

Semiannual Report

Reporting Period: December 1, 1996 to May 30, 1997

Dr. Gregory J. McCarthy

North Dakota State University

Department of Chemistry

Fargo, ND 58102-5516 


\title{
Progress Report, May 30, 1997
}

\author{
DOE Contract DE-FG22-96PC96207
}

\section{Goals and Tasks}

The goals of the project are two-fold: 1) to upgrade semi-quantitative X-ray diffraction (QXRD) methods presently used in analyzing complex coal combustion by-product (CCB) systems, with the quantitative Rietveld method, and 2) to apply this method to a set of by-product materials that have been disposed or utilized for a long period ( 5 years or more) in contact with the natural environment, to further study the nature of CCB diagenesis.

The project is organized into three tasks to accomplish these two goals: 1) thorough characterization of a set of previously analyzed disposed by-product materials, 2) development of a set of CCB specific protocols for Rietveld QXRD, and 3) characterization of an additional set of disposed CCB materials, including application of the protocols for Rietveld QXRD developed in Task 2.

\section{Task 1}

\section{Task Description}

Thorough characterization of a reference set of CCB materials is accomplished by chemical analysis of individual, identifiable mineral grains under scanning electron microscopy with electron probe microanalysis (SEM/EPMA). It serves as a known reference point, for testing the Rietveld method for use with CCB systems (Task 2). This is especially important for parameterization of stoichiometry dependence on Rietveld QXRD solutions, and for generating a generalized set of protocols to follow in the use of the Rietveld method with CCBs. Task 1 is to be completed during study year 1, with SEM/EPMA training under the direction of Dave Brekke (UND-EERC).

\section{Progress Toward Task 1}

An initial reference set of CCB materials was chosen from in-house collections of previously studied materials, for initial analysis sessions, based on mineralogy. Two minerals of particular importance to $\mathrm{CCB}$ diagenesis are ettringite (Et) and thaumasite (Tm). Ettringite, with a nominal stoichiometry of $\mathrm{Ca}_{6} \mathrm{Al}_{2}\left(\mathrm{SO}_{4}\right)_{3}(\mathrm{OH})_{12} * 26 \mathrm{H}_{2} \mathrm{O}$, forms in many high-calcium, high-pH cementitious CCBs as an immediate hydration product. Further hydration and alteration caused by percolating pore solutions leads, in many cases, to significant increases in the amount of ettringite present. Thaumasite (nominal stoichiometry $\mathrm{Ca}_{6} \mathrm{Si}_{2}\left(\mathrm{CO}_{3}\right)_{2}\left(\mathrm{SO}_{4}\right)_{2}(\mathrm{OH})_{12} * 24 \mathrm{H}_{2} \mathrm{O}$ ) has been recognized in deteriorated concrete in contact with gypsum plaster or with sulfate rich ground water, and was first noted to appear in long term CCB disposal situations in our laboratories. The presence of thaumasite is generally coincident with a loss of desirable physical characteristics, including significant loss of compressive strength and increase in permeability. A potentially continuous solid solution exists between these two end-member chemistries, and this solid solution (Et-Tm) has been observed in all CCB systems examined to date. 
Since Rietveld QXRD analysis is performed by refining crystal structures of all known constituent phases, and since variation of chemistry introduced by solid solution will generate differences in the diffraction data being used for these refinements, Et-Tm was chosen as an initial target for Task 1. The seven initial samples include materials known to contain ettringite, thaumasite or intermediate compositions, and are listed in Table 1. A variety of CCB material types is also represented in this set. Samples 970006 and 970007 are from long-term (>10 yrs) disposal material from a western North Dakota utility using high sodium lignite and dry process flue gas desulfurization (FGD). Sample 970008 is from atmospheric fluidized bed combustion (AFBC) residue from the Midwest Grain ethanol plant in Illinois, disposed for 4 years as part of a previous METC study. Material from the Clean Coal Technology limestone injection multistage burner (LIMB) produced in Ohio at the Edison Edgewater power facility, disposed for five years (970010), and also part of previous METC studies, was the final known thaumasite-containing by-product. Additionally, three samples were chosen from a METC co-disposal study involving the Illinois FBC material and Class F fly ash produced at the Duck Creek electric power generating facility. After three years of disposal, no thaumasite formation had been observed in these three co-disposal samples, although abundant ettringite is present.

Two specimens were prepared from each sample. Ground and micronized powder, produced during prior study of each of the samples, was mounted onto double-sided adhesive tape. While these powders were the actual specimens used in prior semi-quantitative QXRD studies, there was some concern about the physical condition and recognizability of the mineral grains following the long grinding times involved in preparing the powders. As such, a second mount was made from unground granular bulk material, which was briefly crushed prior to mounting. The granular material was mounted into conductive carbon paint. All mounts were made on $1.25 "$ carbon blocks, and all specimens were coated with carbon.

Table 1. CCB reference set description

\begin{tabular}{cclcc}
\hline EERC ID & NDSU ID & CCB Material & $\begin{array}{c}\text { Length of } \\
\text { emplacement }\end{array}$ & $\begin{array}{c}\text { Thaumasite } \\
\text { present? }\end{array}$ \\
\hline 970006 & AVS B1 & High Na fly ash / Dry process FGD & $11 \mathrm{yrs}$ & Yes \\
970007 & AVS B4 & High Na fly ash / Dry process FGD & $11 \mathrm{yrs}$ & Yes \\
970008 & 95AugIll 1-2 & AFBC process residue & $4 \mathrm{yrs}$ & Yes \\
970009 & 96MayIll 1-1 & AFBC / Class F co-disposal, well indurated & $3 \mathrm{yrs}$ & No \\
970010 & 94JulyOH & LIMB process residue & $5 \mathrm{yrs}$ & Yes \\
970011 & 96MayIll 1-3 & AFBC / Class F co-disposal, poorly indurated & $3 \mathrm{yrs}$ & No \\
970012 & 96MayIll 2-3 & AFBC / Class F co-disposal, poorly indurated & $3 \mathrm{yrs}$ & No \\
\hline
\end{tabular}

Six of the seven samples were analyzed using SEM/EPMA. Quantitative XRF results were obtained using peak areas and ZAF correction. None of the highly ground specimens were found suitable for reliable analysis. Individual grains were mechanically worn such that mineral assignments based on morphology were not possible. The only grains with distinct crystalline morphology observed in any of the ground specimens were particles of char (only carbon identified by XRF), which were seen in the FBC material. Additionally, the grains were too aggregated to permit analysis of distinct individual grains. Point analyses of aggregates of potentially varying mineralogies have little interpretive value. 
The crushed samples did have identifiable mineral grains. However, disaggregation by mechanical hand grinding was not sufficient to entirely eliminate the potential for problems caused by electron beam focal spots encompassing more than one grain at a time. Preliminary results, as well as training in SEM/EPMA instrumental analytical technique under the direction of an experienced CCB analyst, were obtained on these samples nevertheless. Results of 38 point analyses of suspected Et-Tm phases from six of the eight crushed specimens are presented in Table 2.

Elemental, rather than oxide analyses, were calculated. Water and hydroxyl groups present in the crystal structure of Et-Tm are removed during degassing, making direct interpretation of the elemental results difficult. To account for this, major cations of interest were renormalized versus 6 moles of $\mathrm{Ca}$ (Table 3). With this method, aluminum, silicon, and sulfur stoichiometries in Et-Tm can be read directly (assuming full calcium site occupancy in an ideal crystal). As silicon substitutes for aluminum in the ettringite structure, a charge deficiency develops that must be compensated by oxyanions. Thaumasite nominally has carbonate groups that substitute for the sulfate of ettringite. Plotting sulfur content versus silicon for 32 of the point analyses shows this relationship (Figure 1). A linear regression was performed for the FBC data, with a fairly good fit $\left(\mathrm{R}^{2}=76 \%\right)$. These data show $\mathrm{S}=3.00$ for $\mathrm{Si}=0.00$ and $\mathrm{S}=2.03$ for $\mathrm{Si}=2.00$, as predicted (refer to nominal stoichiometries in earlier discussion).

Figure 1 also shows the range of mutual solubility of aluminum and silicon in Et-Tm seen in CCBs. It should be noted that altered residues from various processes appear to differ markedly in this regard. Since control over disaggregation and grain coatings was not necessarily achieved, these data serve only as preliminary examples of the analyses to be derived. Further study will require suspension and sonication to thoroughly disaggregate the material prior to SEM study.

Complications due to severe flooding of the SEM/EPMA facility located in the UNDEERC in Grand Forks, North Dakota, have rendered immediate further analyses impossible. Repair or replacement of the instruments is now being considered. If desired, updates of estimated return to operation may be provided. If this process takes an unreasonable amount of time, SEM/EPMA equipment is available at NDSU, although use of this equipment was not planned until thorough training at UND-EERC under the experienced direction of Dave Brekke was completed. 
Table 2. Elemental XRF (SEM/EPMA) data

\begin{tabular}{|c|c|c|c|c|c|c|c|c|c|c|c|c|c|c|c|c|c|c|c|}
\hline \multirow{2}{*}{$\begin{array}{c}\text { Sample } \\
\text { NDSU ID }\end{array}$} & \multirow{2}{*}{$\begin{array}{c}\text { Sample } \\
\text { EERC } \\
\text { ID }\end{array}$} & \multirow[b]{2}{*}{$\begin{array}{c}\text { Pt. } \\
\text { Anal. }\end{array}$} & \multicolumn{5}{|c|}{ Atomic mol \% } & \multirow[b]{2}{*}{ Si-K } & \multirow[b]{2}{*}{$\mathrm{Si} / \mathrm{Al}$} & \multirow[b]{2}{*}{$\mathrm{P}-\mathrm{K}$} & \multirow[b]{2}{*}{ S-K } & \multirow[b]{2}{*}{$\mathrm{Cl}-\mathrm{K}$} & \multirow[b]{2}{*}{$\mathrm{K}-\mathrm{K}$} & \multirow[b]{2}{*}{$\mathrm{Ca}-\mathrm{K}$} & \multirow[b]{2}{*}{ Ti-K } & \multirow[b]{2}{*}{$\mathrm{Cr}-\mathrm{K}$} & \multirow[b]{2}{*}{$\mathrm{Fe}-\mathrm{K}$} & \multirow[b]{2}{*}{$\mathrm{Ba}-\mathrm{L}$} & \multirow[b]{2}{*}{$\mathrm{O}-\mathrm{K}$} \\
\hline & & & Phase & $\mathrm{Chi}^{\wedge} 2$ & $\mathrm{Na}-\mathrm{K}$ & $\mathrm{Mg}-\mathrm{K}$ & $\mathrm{Al}-\mathrm{K}$ & & & & & & & & & & & & \\
\hline \multirow[t]{7}{*}{ AVS B1 } & 97006 & a.1a & Afm & 7.56 & 0.0040 & 0.1900 & 3.09 & 1.10 & 0.356 & 0.0000 & 5.76 & 0.0000 & 0.0070 & 10.39 & 0.0040 & 0.0000 & 0.1600 & 0.0043 & 79.30 \\
\hline & & a.2 & & 4.24 & 0.1200 & 0.3600 & 2.82 & 1.52 & 0.539 & 0.0000 & 5.75 & 0.0000 & 0.0069 & 11.18 & 0.0049 & 0.0004 & 0.0081 & 0.0048 & 78.25 \\
\hline & & a.2a & & 2.43 & 0.5600 & 0.4100 & 2.50 & 1.51 & 0.604 & 0.1100 & 4.99 & 0.0089 & 0.0093 & 8.78 & 0.0015 & 0.1000 & 0.1200 & 0.0009 & 80.94 \\
\hline & & a. $2 b$ & & 2.71 & 1.5800 & 1.6500 & 2.76 & 4.03 & 1.460 & 0.0069 & 6.19 & 0.0900 & 0.0097 & 10.49 & 0.0017 & 0.1000 & 0.4700 & 0.1500 & 72.47 \\
\hline & & a.3b & & 10.71 & 0.0050 & 0.1700 & 3.30 & 0.87 & 0.264 & 0.0030 & 5.51 & 0.0090 & 0.0086 & 10.31 & 0.0024 & 0.0001 & 0.0090 & 0.0020 & 79.84 \\
\hline & & a.3c & & 5.73 & 1.3600 & 1.2100 & 2.71 & 5.15 & 1.900 & 0.0080 & 5.37 & 0.2000 & 0.0000 & 8.83 & 0.0010 & 0.0090 & 0.3200 & 0.1200 & 74.73 \\
\hline & & a.3d & & 3.73 & 1.9100 & 1.8700 & 2.82 & 4.44 & 1.574 & 0.0080 & 4.98 & 0.0010 & 0.0000 & 7.47 & 0.0000 & 0.1200 & 0.1600 & 1.6000 & 76.07 \\
\hline \multirow[t]{9}{*}{ 95Auglll1-2 } & 970008 & a.1 & Afm & 7.59 & 0.2400 & 0.8200 & 0.93 & 2.98 & 3.204 & 0.1200 & 3.60 & 0.0081 & 0.0058 & 10.46 & 0.0047 & 0.0006 & 0.0085 & 0.0046 & 80.86 \\
\hline & & a.2 & & 3.31 & 0.0010 & 0.0050 & 0.45 & 3.24 & 7.200 & 0.0030 & 3.88 & 0.0080 & 0.0050 & 10.23 & 0.1100 & 0.0000 & 0.3900 & 0.0040 & 81.69 \\
\hline & & a.3 & & 3.49 & 0.0000 & 0.0040 & 3.14 & 0.40 & 0.127 & 0.0010 & 4.32 & 0.0900 & 0.0064 & 9.52 & 0.0040 & 0.0001 & 0.1700 & 0.0038 & 82.35 \\
\hline & & a.4 & & 5.12 & 0.0080 & 0.0090 & 1.83 & 2.43 & 1.328 & 0.0030 & 4.43 & 0.0900 & 0.0078 & 11.28 & 0.0026 & 0.0002 & 0.0092 & 0.0022 & 79.94 \\
\hline & & a. 6 & & 8.24 & 0.0070 & 0.0700 & 1.88 & 2.55 & 1.356 & 0.0037 & 4.78 & 0.0082 & 0.0077 & 11.80 & 0.1100 & 0.0700 & 0.0091 & 0.0023 & 78.73 \\
\hline & & a.7 & & 11.99 & 0.1900 & 1.2300 & 2.60 & 2.15 & 0.827 & 0.0800 & 5.37 & 0.0900 & 0.0060 & 12.59 & 0.0052 & 0.0005 & 0.0079 & 0.0053 & 75.69 \\
\hline & & a.8 & & 2.11 & 0.0060 & 0.1100 & 2.24 & 2.51 & 1.121 & 0.0036 & 5.11 & 0.1200 & 0.0600 & 12.48 & 0.0028 & 0.0001 & 0.1000 & 0.0025 & 77.26 \\
\hline & & a.9 & & 1.7 & 0.0000 & 0.3400 & 3.44 & 0.32 & 0.093 & 0.0010 & 4.91 & 0.0090 & 0.0070 & 9.39 & 0.0030 & 0.0090 & 0.5600 & 0.0032 & 81.04 \\
\hline & & a.10 & & 3.06 & 0.2400 & 0.0090 & 1.56 & 2.26 & 1.449 & 0.0030 & 4.36 & 0.0070 & 0.0070 & 11.77 & 0.0028 & 0.1100 & 0.1500 & 0.0024 & 79.54 \\
\hline \multirow[t]{10}{*}{ 94JulOH1-2 } & 970010 & a.1 & & 11.4 & 0.3700 & 0.0040 & 0.84 & 3.29 & 3.917 & 0.0030 & 3.53 & 0.0080 & 0.0060 & 9.79 & 0.0040 & 0.0006 & 0.0086 & 0.0044 & 82.17 \\
\hline & & a.1a & & 2.91 & 0.0000 & 0.2500 & 1.47 & 2.69 & 1.830 & 0.0040 & 4.01 & 0.0900 & 0.0080 & 9.86 & 0.0020 & 0.0098 & 0.2100 & 0.0800 & 81.35 \\
\hline & & a. $1 b$ & & 1.72 & 0.0000 & 0.2800 & 2.08 & 5.77 & 2.774 & 0.0010 & 9.14 & 0.0070 & 0.0020 & 32.13 & 0.0060 & 0.0000 & 0.9400 & 0.0060 & 49.64 \\
\hline & & a. $1 \mathrm{c}$ & & 2.16 & 0.0040 & 0.0020 & 2.40 & 2.83 & 1.179 & 0.0040 & 5.23 & 0.0070 & 0.0080 & 12.92 & 0.0010 & 0.0090 & 0.4400 & 0.0011 & 76.18 \\
\hline & & a.1d & & 1.69 & 0.1900 & 0.1100 & 1.39 & 3.62 & 2.604 & 0.0040 & 4.24 & 0.0070 & 0.0070 & 13.26 & 0.0020 & 0.1400 & 0.2800 & 0.0024 & 76.76 \\
\hline & & a.1e & & 5.72 & 0.0090 & 0.0900 & 1.10 & 3.56 & 3.236 & 0.0500 & 4.23 & 0.0081 & 0.0081 & 11.22 & 0.0025 & 0.0002 & 0.0093 & 0.0020 & 79.74 \\
\hline & & a.2a & & 2.33 & 0.1300 & 0.2400 & 1.96 & 3.46 & 1.765 & 0.0040 & 5.06 & 0.0070 & 0.0060 & 15.62 & 0.0030 & 0.0090 & 0.5900 & 0.1000 & 72.83 \\
\hline & & a. $2 b$ & & 2.58 & 0.0020 & 0.2300 & 2.61 & 3.95 & 1.513 & 0.1000 & 5.25 & 0.1000 & 0.0070 & 15.06 & 0.0030 & 0.0001 & 0.1900 & 0.0030 & 72.51 \\
\hline & & a.2c & & 2.08 & 0.0070 & 0.1900 & 3.26 & 3.26 & 1.000 & 0.0060 & 3.65 & 0.0070 & 0.0080 & 10.77 & 0.0003 & 0.0076 & 2.2600 & 0.1100 & 76.50 \\
\hline & & a.3a & & 3.67 & 0.3600 & 0.3500 & 0.72 & 2.99 & 4.153 & 0.0030 & 3.50 & 0.0080 & 0.0060 & 9.14 & 0.0040 & 0.0000 & 0.0087 & 0.0041 & 82.95 \\
\hline 96MayllI2-3 & 970011 & a.1a & & 3.32 & 0.0070 & 0.0030 & 2.09 & 2.49 & 1.191 & 0.1100 & 4.06 & 0.0080 & 0.0052 & 11.54 & 0.0046 & 0.0003 & 0.1200 & 0.0900 & 79.49 \\
\hline & & a. $1 b$ & & 5.62 & 0.0030 & 0.0020 & 0.37 & 1.83 & 4.946 & 0.0010 & 0.70 & 0.0800 & 0.1400 & 20.42 & 0.0053 & 0.0011 & 0.1400 & 0.0054 & 76.33 \\
\hline & & a.1c & & 2.67 & 0.6500 & 0.6400 & 11.67 & 12.07 & 1.034 & 0.0081 & 1.85 & 0.0600 & 0.5500 & 8.31 & 0.1800 & 0.0084 & 0.4000 & 0.0900 & 63.53 \\
\hline & & a.1d & & 2.96 & 1.5400 & 0.9000 & 8.59 & 20.16 & 2.347 & 0.0050 & 2.16 & 0.1300 & 1.6600 & 6.82 & 0.0096 & 0.1200 & 2.1000 & 0.0087 & 55.82 \\
\hline 96Maylll1-3 & 970012 & a. 1 & & 5.84 & 0.0080 & 0.1300 & 1.69 & 2.46 & 1.456 & 0.0020 & 3.83 & 0.0080 & 0.1100 & 10.16 & 0.0043 & 0.1600 & 0.0084 & 0.0043 & 81.46 \\
\hline & & a.2 & & 6.75 & 0.0060 & 0.4900 & 2.02 & 1.26 & 0.624 & 0.2500 & 3.42 & 0.0070 & 0.0900 & 7.21 & 0.0000 & 0.0080 & 0.7500 & 0.0098 & 84.51 \\
\hline
\end{tabular}




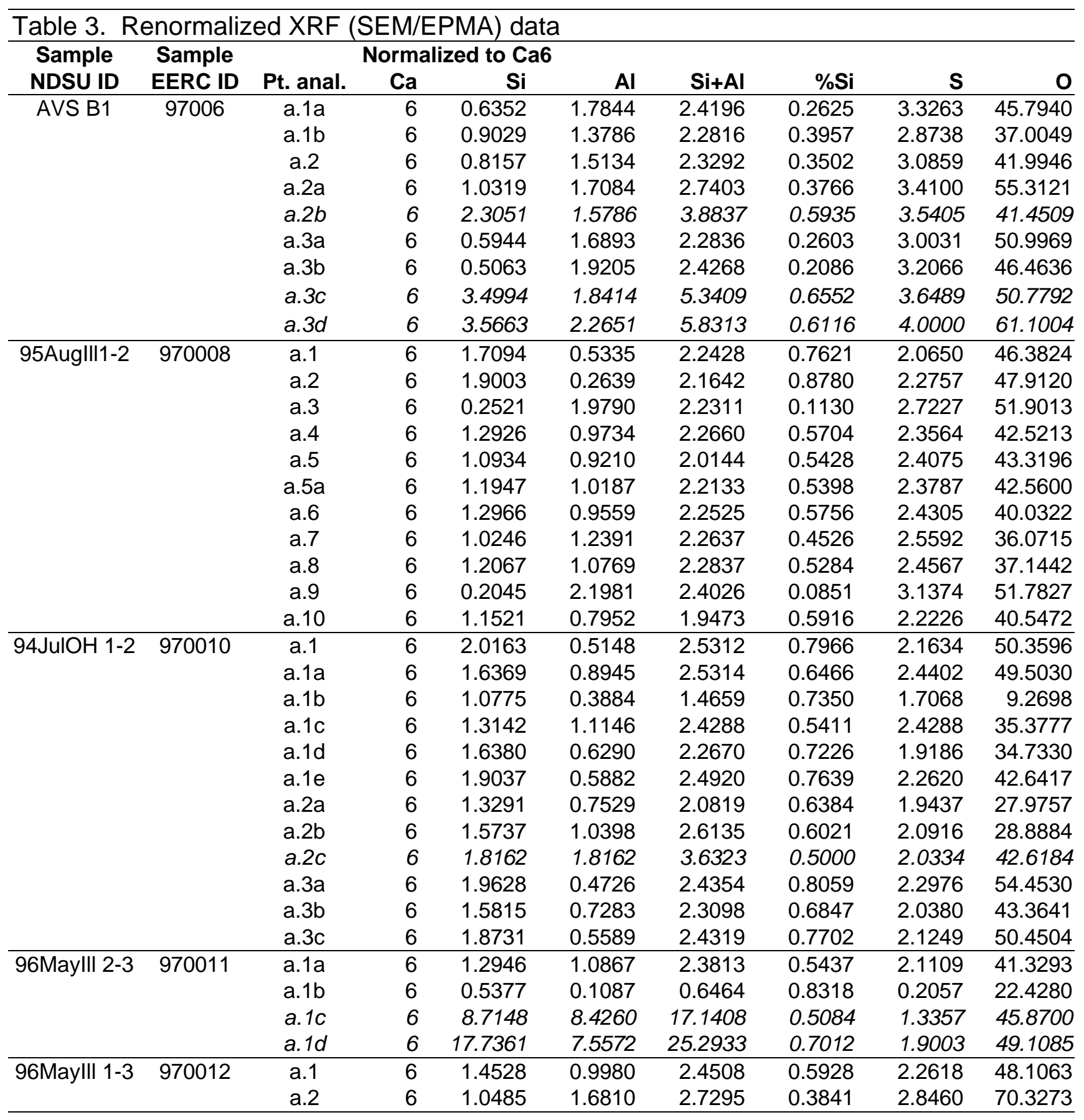


Figure 1.

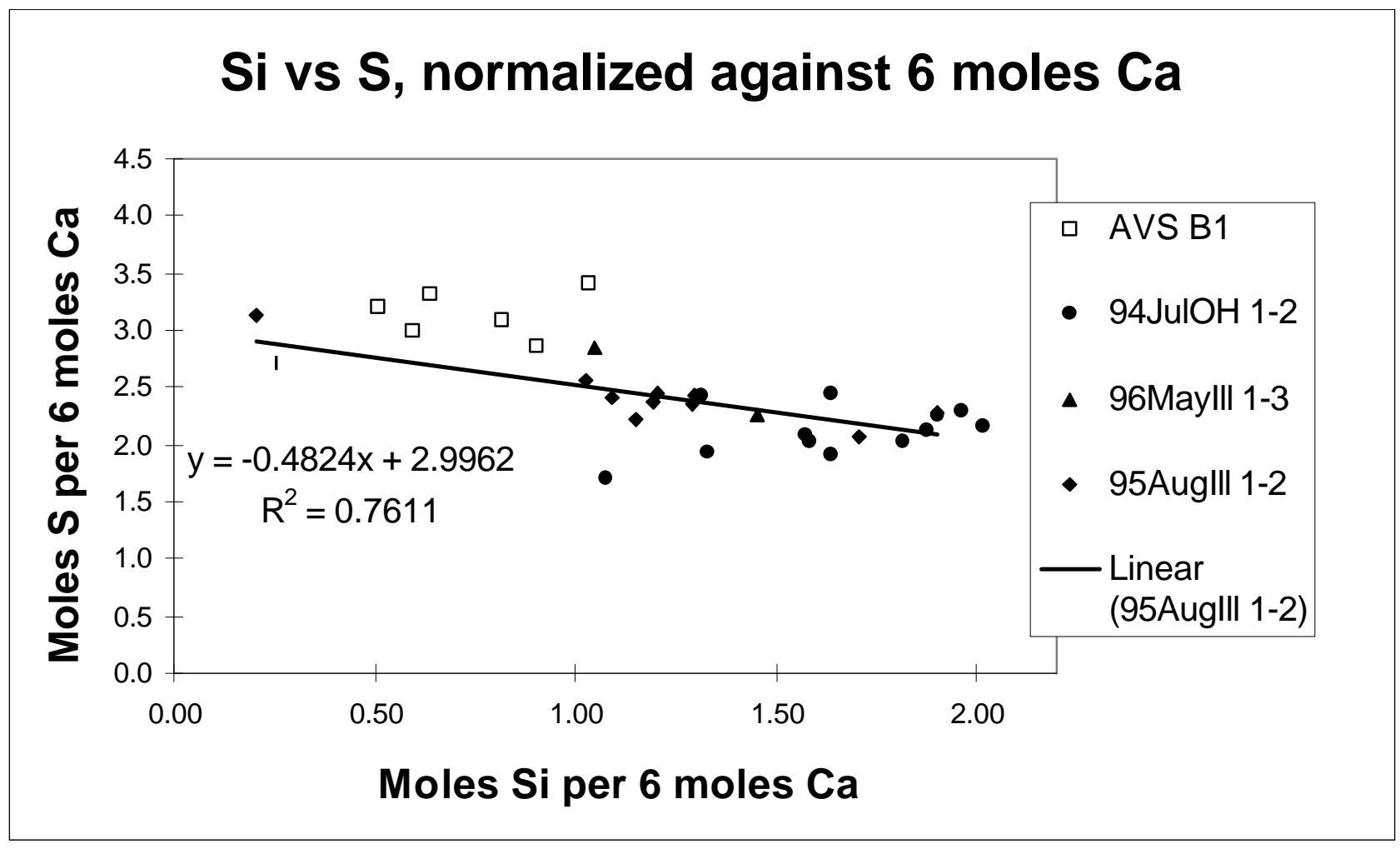

Task 2

\section{Task Description}

Task 2 is composed of 3 major steps 1) create a database of relevant phases, 2) determine a standard set of procedures for the refinement of coal-combustion by-products (CCBs) to be used in Rietveld refinements, and 3) create a Web page to make this information accessible to the research community.

\section{Progress Toward Task 2}

Step 1.

The first step in Task 2 of the proposal is to create a database of the relevant phases. This database will be accessible to the research community through the Web page described in the next section. The mineral phases will be accessible through the .exp files for the Rietveld program, GSAS. The phases contained in these files can be easily read directly into the working .exp file for a GSAS refinement.

A literature search is being done on each of the phases, starting with the Inorganic Crystal Structure Database (ICSD), to find many of the pertinent references. Searches of the CarlUncover database and Current Contents are also being done to determine if there are any new papers on the structures of these phases.

Once a number of references for a phase has been obtained, the programs POWD and $\underline{\text { DINT }}$ are run on each structure. The output from DINT is used to determine if there are any discrepancies such as unusual bond lengths or bond angles. The output from POWD is used to 
compare the diffraction patterns of the structures. This step narrows down the number of possible structures for a given phase to typically not more than five structures.

The next step for determining the structures that will go into the database is to use the structure in an actual refinement. To get to this point, the structures must be reported in the literature with isothermal parameters (there may be some exceptions, if necessary), few omissions of atoms in the structure (hydrogens are the exception) and have reasonable output from POWD and DINT.

The database will then consist of one of the following two choices:

1. One .exp file for each phase to be calculated, with the individual structures contained in the file as separate phases.

2. A .exp file for each of the structures will be created, with the recommended phase being $\boldsymbol{x} \boldsymbol{x} \boldsymbol{x} \boldsymbol{x} 1 . \exp$, the next most reliable being $\boldsymbol{x} \boldsymbol{x} \boldsymbol{x} \boldsymbol{x} 2 . \boldsymbol{e x p}$, etc.

These .exp file will then be accessible for downloading through our Web page, as described in the next section. A report of the progress towards completion of the database is attached to the end of this report.

Step 2.

The second step towards completing Task 2 is the determination of a standard set of refinement procedures. The first requirement of this step was to determine how GSAS works with respect to various complications that will be encountered in the CCB samples. A set of NIST standards was used to determine the effects of preferred orientation, phase abundance and absorption effects. Samples from the lab of Dr. Philip Boudjouk (NDSU Chemistry) have been used to examine solid solution effects. Amorphous content has yet to be examined.

The next requirement is obtaining CCB samples (or diffraction patterns) to determine a set of refinement procedures for these samples. There will be a set of cases for which refinement procedures will be determined, such as percent quartz (or some other mineral), full phase analysis, quick phase analysis, and partial phase analysis. For each of the cases the following will be determined:

1. The refinement procedure for that case.

2. The limits of the method.

3. The errors associated with the method.

These will be determined by doing a comprehensive study on each method using samples with known composition.

Step 3.

The final step in Task 2 is the creation of a Web page for easy access to the results of our research. This Web page will contain the structure information and the standard refinement procedures discussed in the preceding sections, as well as information about our laboratory and the persons responsible for the research. To see what the Web page will look like go to the URL

http://quantum.chem.ndsu.nodak.edu/ winburn/index.html.

The sections of the most concern are those containing the phase information and the refinement procedures. These two sections will be addressed here. First, the section containing the phase information will be discussed, followed by the section on the refinement procedures.

The section containing the phase information will contain links to each of the .exp files that will be stored on the Web server. The researcher will then be able to download the files of 
interest to him or her by right clicking on the phase of interest and choosing Save Link As or Save As on the menu that appears. The files will then be saved on their computer and will be available to be included in their $\exp$ files. A Readme.doc file will also be included in this section. The Readme.doc file will contain information on how to download the .exp files, as well as information on how to read the .exp files into their working .exp files.

The section containing the refinement procedures will have files that can be downloaded for various levels of use. The files will contain information on the ideal scan parameters for the method, the steps in the refinement, errors associated with the method, and levels of accuracy expected with this method. Examples of methods that may be included are quick scans for determination of percent quartz, full quantitative analysis of a complex system, or partial phase analysis (ratios) of a simple system.

This section will also have a Readme.txt file that will explain the details of each of the methods and how to download them. The Readme.txt file will explain how to download the procedure files or how to print them directly from the Web page.

The Web page may or may not have a section with Test Mixtures, . asc files of powder scans that would be posted. These scans can be of the NIST standard mixtures or of CCB mixtures. This is something that must be discussed in the near future. This section would also have a read.me file. This read.me file would once again contain directions for downloading files from the Web page and would also include information about each of the test mixtures, instrumental configuration and scan parameters. Another point to discuss is possibly two separate read.me files, one with the percentages of each phase and a second without this information.

\section{Summary of Task 2}

\section{Step 1}

Progress:

The database of phases is well underway, with the majority of the phases having at least two structures to work with. The majority of the phases have had their structures undergo POWD and DINT calculations. Approximately one half of the phases have their structures inputted into .exp files. Powder patterns have been obtained for 12 phases. We have a limited number of samples of the pure phases.

Need to do:

The remainder of the structures must be transferred into .exp files. The final cut of structures must be determined. Powder patterns need to be obtained for the remainder of the phases. Samples of each phase should be obtained.

\section{Step 2}

Progress:

The NIST standards have been thoroughly studied. Solid solution behavior has also been studied through the use of samples from the Boudjouk lab.

Need to do:

Study amorphous samples. Obtain powder patterns for previously studied CCB samples and samples that have been prepared in the lab using pure phases. Determine the specific methods to be included on the Web page and determine the procedure, limitations and errors for each method. 
Step 3

Progress:

A Web page has been started and can be linked to the appropriate Web page at any time. Need to do:

Add links to the Web page as they become available.

\section{Task 3}

\section{Task Description}

Task 3 involves study of an additional set of disposed CCBs, including SEM/EPMA and detailed qualitative XRD analysis, as well as Rietveld QXRD application, based on knowledge and protocols gained in Task 2. Two core samples have been secured, and three additional cores are being sought. Agreements for coring permission and support for Task 2 are to be obtained by the end of year 2 of the grant period, with core analyses to be completed by the end of year two.

\section{Progress Toward Task 3}

Approximately 50 feet of core have been recovered from the landfill of a Minnesota utility burning Wyoming and Montana coals, and using dry process flue gas desulfurization. This material ranges in age from as much as five years old to recently deposited. The core is in cold storage at NDSU, and will be analyzed during the coming summer. Core material recovered from a road base embankment in Illinois has also been secured, and will also be studied during the 1997 summer. The material was produced by circulating fluidized bed combustion (CFBC), and has been emplaced for up to three years. Detailed histories of ash analysis and emplacement exist for each of these two sites, allowing for potential correlation with original bulk chemistry.

A verbal agreement for sampling of cementitious North Dakota fly ash buried up to 18 years has been obtained. Much of this ash has been studied extensively (as collected, prior to burial) in our laboratories.

Sampling of Class C fly ash in Illinois and Class F fly ash in Kentucky are under negotiation. Additional coring of long term disposal sites is being sought in North Dakota for FGD and FBC materials.

ref: xtalllc:Idean\fetcldoe97rp1.doc 